\title{
El contexto como una estrategia para fomentar el sentido de utilidad de las Matemáticas en estudiantes de ciencias sociales
}

\author{
Maritza Peña-Becerril( ${ }^{(1,2)}$ y Claudia Camacho-Zuñiga ${ }^{(1,2) \star}$ \\ (1) Escuela de Ingeniería y Ciencias, Tecnológico de Monterrey, Ave. Eduardo Monroy Cárdenas 2000, Toluca 50110, \\ Edo. de México, México. (e-mail: maritza@tec.mx) \\ (2) Tecnológico de Monterrey, Writing Lab, TecLabs, Vicerrectoría de Investigación y Transferencia de Tecnología, \\ Monterrey 64849, NL, México. (e-mail: claudia.camacho@tec.mx)
}

${ }^{*}$ Autor a quien debe ser dirigida la correspondencia.

Recibido Jul. 10, 2019; Aceptado Sep. 3, 2019; Versión final Oct. 7, 2019, Publicado Feb. 2020

\begin{abstract}
Resumen
Se diseñó una actividad de aprendizaje para el tema funciones y derivadas contextualizada en las temáticas corrupción y contaminación del agua, la cual se contrastó contra una actividad tradicional. Esto se hizo como una herramienta para fomentar el interés y el sentido de utilidad hacia las matemáticas en universitarios de primer año de áreas de ciencias sociales. Los estudiantes que realizaron la actividad poseían un incipiente sentido de utilidad de las matemáticas y su interés se centraba en problemáticas sociales, económicas y medioambientales. Se concluyó que el contexto sí propició un mejor desempeño de los alumnos en aquellas preguntas relacionadas con interpretación de conceptos, mientras que no mostró beneficios significativos en las que requerían repetición de algún algoritmo. Finalmente, los estudiantes percibieron con mayor gusto, interés y sentido de utilidad por la actividad contextualizada, por encima de la tradicional, fomentando en mayor medida su competencia matemática.
\end{abstract}

Palabras clave: matemáticas; actividad contextualizada; percepción de estudiantes; sentido de utilidad; innovación educativa.

\section{Context as a strategy to promote the perceived usefulness of Mathematics in social science students}

\begin{abstract}
A learning activity was designed for the topic functions and derivatives contextualized in the themes of corruption and water pollution, which was contrasted against a traditional activity. This was done as a tool to promote interest and usefulness sense of mathematics in first-year social science undergraduate students. The students who participated in the activity had an incipient perceived usefulness of mathematics and their interest was focused on social, economic and environmental issues. It was concluded that the context provoked a better performance of the students in those questions related to interpretation of concepts, while it did not show significant benefits in those that required the repetition of an algorithm. Finally, the students perceived the contextualized activity with greater pleasure, interest and usefulness sense over the traditional one, improving to a greater extent their mathematical competence.
\end{abstract}

Keywords: mathematics; contextualized activities; students' perceptions; perceived usefulness; educational innovation 


\section{INTRODUCCIÓN}

Las Matemáticas hoy en día tienen un rol no solo relevante sino también creciente (Gravemeijer et al., 2017). A nivel internacional, tanto las universidades como las instituciones gubernamentales son conscientes de su importancia, y la de otras áreas STEM (Ciencia, Tecnología, Ingeniería y Matemáticas, por sus siglas en inglés), para el bienestar económico y la calidad de vida de su población (Reid et al., 2005). Sin embargo, esta concepción no es siempre compartida por los estudiantes, pues junto con Física y Química, las Matemáticas son las asignaturas que ellos más rechazan (Serrano et al., 2008; Osborne et al., 2003). La esencia del rechazo a las Matemáticas se debe al notable y recurrente fracaso de los alumnos a lo largo de su vida académica (Serrano et al., 2008); sin embargo, la problemática no sólo radica en la capacidad o incapacidad de los estudiantes para comprender determinados temas, sino en su actitud hacia esta ciencia (Morales y García, 2013; Ayob y Yasin, 2017). Gran parte del alumnado llega a esta asignatura condicionando su punto de vista emocional que se traduce desde un letargo o apatía al realizar las actividades de aprendizaje (Morales y García, 2013), hasta una baja autoconfianza e independencia durante la resolución de problemas. Esto causa preocupación ya que los estudiantes con mejores actitudes hacia las Mat. denotan motivaciones intrínsecas relacionadas con su aprendizaje (Perry, 2011, citado por Palacios et al., 2014; Wood et al., 2012; Farias y Pérez, 2010). Lo cual, se refleja en numerosos estudios (Zainal et al., 2017; Wan y Quiping, 2015; Altawallbeh et al., 2015, citados por Ayob y Yasin, 2017; Morales y García, 2013) que han demostrado una correlación significativa entre las actitudes del estudiante y el rendimiento académico.

La actitud hacia las Matemáticas está relacionada con el gusto, la valoración y el aprecio por la disciplina como menciona Jurdak (2006, citado por Beswick, 2011). La valoración es lo que Morales et al. (2010) llaman reconocerla como una fuente de conocimiento y aprendizaje. Mientras que para Wood et al. (2012) las percepciones de esta disciplina son parte de una jerarquía de tres niveles (del más amplio al más limitado): 1) Las Mat. como una aproximación hacia la vida y como una forma de pensar; 2) Las Mat. consisten en construir y utilizar modelos, tanto específicos de ciertos aspectos de la realidad como abstractos de estructuras lógicas y 3) Las Mat. como una herramienta de componentes y procedimientos. En general, se considera que los estudiantes con percepciones más amplias hacia las Matemáticas desarrollan actitudes positivas hacia ellas, llevándolos a mostrar mejores desempeños. Por el contrario, estudiantes con percepciones más limitadas a menudo muestran actitudes negativas, las cuales no solo afectan su desempeño sino también su aprendizaje (Ayob y Yasin, 2017). En este caso, el problema surge cuando los estudiantes no la perciben como una herramienta y por lo tanto, los conocimientos que puedan adquirir se vuelven inertes (Gutiérrez, 1999; Serrano et al., 2008).

Según Perry (2011, citado en Palacios et al., 2014) los estudiantes con mejores actitudes hacia las Matemáticas tienen más altas percepciones de utilidad de las mismas. Es decir, las reconocen como una herramienta útil para ellos. Y cuando esto ocurre, los alumnos saben no solo lo que es, sino cuándo y cómo usarlas (Serrano et al., 2008). Esto también coincide con la afirmación de Skovsmose (1999, citado por Sáenz y García, 2015) al mencionar que las Mat. como una herramienta para la resolución de problemas son Matemáticas en acción. Desde esta perspectiva, el alumno puede hacer uso de ellas para descubrir, explorar, comprender y finalmente, desarrollar una conciencia de su utilidad y beneficios para la sociedad; tanto en su entorno más inmediato, como en el mundo global. Esto se refleja directamente en la definición de competencia; ser competente, en Matemáticas, va más allá de ser capaz de realizar cálculos. Ser competente significa poseer la capacidad para identificar y entender el rol que juega esta ciencia en el mundo (PISA, 2003, citado por Morales et al., 2010; Zemelman et al., 1998, citado por Farias y Pérez, 2010). Además, implica emitir juicios bien fundamentados y utilizar los conocimientos para satisfacer una necesidad como ciudadano constructivo, comprometido y reflexivo (PISA, 2003, citado por Morales et al., 2010).

Es ahí donde la labor del docente es indispensable, ya que su tarea fundamental es fortalecer la competencia matemática de sus alumnos (Ruiz, 2001), debiendo entonces posicionarla y descubrir su valor ante los estudiantes. No basta explicar bien la materia y asegurarse que aumenten sus conocimientos matemáticos (Morales y García, 2005). Se requiere cambiar su percepción del sentido de utilidad (Beswick, 2011; Palacios et al., 2014), llevándolos a valorar esta ciencia. Las aplicaciones de las Matemáticas en el actual mundo globalizado y dominado por la economía del conocimiento son numerosas. Incluso en el ámbito escolar, esta asignatura tiene relación con muchas áreas del conocimiento, brindando múltiples oportunidades para ejemplificar su aplicación a todos los niveles educativos. Esto hace injustificable estudiarla como una ciencia aislada; pues si eso sucede, se vuelven inútiles y sin ninguna posibilidad de ser atractivas para los estudiantes (Sáenz y García, 2015). La clase de Matemáticas llega a ser árida si sólo se centra en la explicación de algoritmos o conceptos. Por el contrario, si este conocimiento se les presenta aplicado, entonces tiene sentido para los estudiantes, llegando incluso a generar interés cuando repercute en su cotidianidad inmediata (Sáenz y García, 2015). Azcárate et al. (2015) mencionan que la enseñanza y el aprendizaje de las Mat. no debe reducirse a una simple acción de poner en escena símbolos, definiciones y teoremas, porque esto es lo que lleva a no tener sentido para el estudiante. Tampoco debe esperarse a que los estudiantes "adquieran 
experiencia" o a que en el futuro entiendan el propósito de la materia. El interés y motivación por el estudio de los temas vistos en cada lección, deben atraer al alumno mientras cursa los estudios (Kline, 1986). Pues la motivación moldea la forma de pensar del alumno y, con ello, condiciona el tipo de aprendizaje resultante (Farias y Pérez, 2010).

El currículo de Matemáticas, especialmente a nivel superior, a menudo es establecido por expertos en la disciplina, enfocados en los aspectos técnicos de la materia más que en las conexiones con sus aplicaciones y el uso en la vida profesional (Reid et al., 2005). Estos currículos hacen complicado construir al interior del aula pensamientos matemáticos y lograr que los estudiantes vean la relevancia de esta ciencia para sus propios estudios, profesiones y situaciones reales. Sin embargo, los resultados de las investigaciones exigen hacer estos conocimientos más ligados a la experiencia, incluyendo la aplicación de los principios de realidad, necesidad y utilidad (Farias y Pérez, 2010; Morales y García, 2013); y así, desarrollar actividades y materiales de aprendizaje involucrando a los estudiantes con una noción de aprender Mat. con un propósito más allá de aprobar el curso y adquirir algunas técnicas específicas (Reid et al., 2005). Gravemeijer et al. (2017) incluso afirman que uno de los propósitos de la educación matemática debe ser preparar a los estudiantes para su desempeño laboral. Con ello se refieren a la aplicación de actividades con problemas auténticos de su área profesional, mostrando a la Mat. como un conocimiento útil y balanceando las formas ortodoxas y no ortodoxas de la enseñanza de esta asignatura.

En esta dirección se encuentran la Educación Matemática Realista (RME, por sus siglas en inglés) y la Educación Matemática Crítica (EMC). La primera utiliza los problemas contextuales para mostrar la naturaleza de las Mat. como una actividad humana que se debe abordar mediante el aprender haciendo y, en la cual los contextos no están restringidos al mundo real o a la vida cotidiana, pueden ser incluso fantasiosos con la condición de ser reales en la mente de los alumnos (Dickinson y Hough, 2012; Cooper y Harries, 2002, citado por Beswick, 2011). En cuanto a la EMC, como lo menciona Sáenz y García (2015), se interesa por temas como la equidad, la inclusión/exclusión, la justicia social y ha dado lugar a diversas teorías que progresivamente se van acercando a estructuras explicativas de corte sociopolítico y cultural, como una herramienta interpretativa del aprendizaje de las Mat. Esta perspectiva ofrece diversas opciones en la aplicación del conocimiento, marcando la pauta y brindando los parámetros para poder incluir el análisis de la corrupción, la pobreza, la desigualdad social, el hambre, la contaminación, etc., como temas centrales de estudio. Con este enfoque el alumno estará aprendiendo, practicando y haciendo Mat. mientras genera conciencia, valores y herramientas para comprender su entorno.

Dado que hoy en día la importancia y las aplicaciones de las Matemáticas también conciernen a áreas más allá de los campos STEM, tales como las Ciencias Sociales, las Finanzas, la Logística y el Análisis de riesgo (Gravemeijer et al., 2017), las universidades han acertado incluyendo su estudio en estas carreras profesionales. Sin embargo, Osborne et al. (2010) señalan que una tendencia reciente es elegir carrera profesional para huir de las Mat. Lo cual a su vez explica el porqué los alumnos de las áreas de Ciencias Sociales muestran una mayor apatía que los estudiantes de áreas STEM, tal como lo han percibido las autoras en sus más de 20 años de experiencia docente en esta asignatura a nivel superior.

En este marco de referencia se plantea la presente investigación, donde se analiza el desempeño y la percepción de los estudiantes de Ciencias Sociales de las Matemáticas y se comparan dos actividades de aprendizaje: la primera bajo el enfoque tradicional (AT) y la segunda contextualizada (AC) con el criterio de EMC. El objetivo es analizar una actividad contextualizada de aprendizaje como una herramienta para fomentar el interés, el gusto y el sentido de utilidad hacia las Matemáticas escolares, teniendo como preguntas de investigación: 1) ¿Cuáles son las percepciones del sentido de utilidad de las Matemáticas escolares de los estudiantes que cursan Matemáticas para Ciencias Sociales? 2) ¿Cómo una actividad de aprendizaje de Matemáticas contextualizada en problemas sociales comparada con una tradicional, impacta el desempeño, el sentido de utilidad y la percepción de aprendizaje de los estudiantes?

\section{METODOLOGÍA}

La presente investigación es un estudio cuantitativo de tipo descriptivo y correlacional. Lo primero se debe a la necesidad de especificar las características y propiedades del grupo de trabajo para su análisis. Lo segundo, por el propósito de medir el grado de relación entre las variables de estudio -interés y gusto por las actividades didácticas y sentido de utilidad de las Matemáticas (Hernández et al., 2014). El estudio es no experimental del tipo transversal.

La población estudiantil en esta investigación es mixta con edades entre 18 y 20 años perteneciente al área de Ciencias Sociales (CS), de licenciaturas como: Derecho, Creación y desarrollo de empresas, Negocios Internacionales, Economía, Administración de empresas, Contaduría pública y Finanzas, Comunicación y Medios digitales y Mercadotecnia. De acuerdo al plan de estudios del ITESM, Matemáticas I para CS es un 
curso obligatorio para todas las carreras profesionales de la Escuela de Negocios y Humanidades (ENH), y la Escuela de Ciencias Sociales y Gobierno (ECSG), en la que se trata Cálculo diferencial de una variable, abordando los temas de funciones lineales y no lineales, límites, continuidad y derivadas. Matemáticas II es un curso de Cálculo en una y varias variables y Álgebra lineal, cursada solo por algunas carreras profesionales de la ENH. En ésta se abordan los temas de integración definida e indefinida de una variable, derivadas parciales y optimización de funciones en varias variables, finalizando con matrices, determinantes y resolución de sistemas de ecuaciones.

\section{Percepciones del sentido de utilidad de las Matemáticas}

Según la Real Academia Española, útil es un adjetivo otorgado a aquello "[q]ue puede servir y aprovechar en alguna línea" o "[q]ue trae o produce provecho, comodidad, fruto o interés". En esta connotación, útil es una cualidad que otorga un sujeto a un sustantivo (objeto directo) en una línea de interés (objeto indirecto). En este trabajo, "sentido de utilidad" refiere a aquellos casos donde el propio sujeto constituye la línea de interés, es decir: "yo creo que es útil para mi" o "me es útil". Para abordar la primera pregunta de investigación se realizó un estudio exploratorio aplicando dos encuestas en distintos momentos. La primera, titulada "Las Matemáticas en tu vida", con cuatro preguntas cerradas con respuestas delimitadas fue aplicada a 91 estudiantes inscritos en las materias Matemáticas I y II para CS, en el semestre Agosto-diciembre 2016, con la intención de conocer su sentido de utilidad de las Mat.

Farías y Pérez (2010) recomiendan conocer las necesidades y expectativas de los estudiantes para luego guiar apropiadamente el aprendizaje. Con esta intención, la segunda encuesta, titulada "Temas de interés", diseñada y analizada con la técnica análisis del discurso, se aplicó el primer día de clases del semestre Eneromayo 2017, a los 100 alumnos inscritos en las materias antes mencionadas. Dicha encuesta estuvo integrada por dos preguntas abiertas: Pregunta 1) “¿Qué problema quieres resolver?”, acompañado de la siguiente instrucción verbal: "Si en este momento tuvieras todo el conocimiento y poder necesario, escribe una problemática que quisieras ver resuelta." Pregunta 2) "¿En qué te ayudan las Matemáticas para abordar y resolver ésta problemática?", con la siguiente instrucción verbal: "Describe la forma en la que las Mat. te ayudan a resolver la problemática que planteaste en la Pregunta 1". Los datos obtenidos se analizaron mediante estadística descriptiva.

\section{Desempeño y percepciones en una actividad de aprendizaje contextualizada}

Para hallar la respuesta a la segunda pregunta de investigación, se aplicaron, en el semestre Enero-mayo 2017, dos actividades de aprendizaje a los alumnos inscritos en Matemáticas I para CS. Ambas actividades abordan el tema "Funciones y derivadas": una con enfoque tradicional (AT), adaptando ejercicios del libro de texto sugerido en el programa analítico de la materia y otra con enfoque EMC, siendo ésta una actividad contextualizada (AC). El contexto de esta última se seleccionó entre las respuestas a la encuesta "Temas de interés", eligiendo Corrupción y Contaminación del agua. Las actividades de aprendizaje se muestran en las Figuras 1 y 2 , respectivamente.

Instrucciones: Desarrolle cada uno de los problemas siguientes. Cuide que su procedimiento sea claro y ordenado. Enmarque sus resultados finales.

1. Sea la función $\square(\square)=3 \square^{2}+6 \square+1$,

1.1 Evalúe: $\square(3), \square(-2), \square(5) \square \square(0)$.

1.2 Ubique en un plano cartesiano los puntos anteriores y bosqueje el comportamiento de $\square$ ( $\square$ )en todo su dominio

2. Para las coordenadas siguientes:

$(1,5),(2,-1),(3,2),(4,3),(5,7),(6,7),(7,0),(8,2),(10,-1),(11,3) \square(13,1)$

2.1 Halle un modelo de función, utilizando cualquier software, que contenga el menor error de aproximación y llámele $\square(\square)$.

2.2 Determine con su modelo $\square(14)$

2.3 Calcule la primera derivada del modelo obtenido en 2.1 , determine el resultado de $\square$ '(3), $\square$ '(0), $\square^{\prime}(2)$ e interprételos.

2.4 ¿A qué velocidad cambió $\square(\square)$ en $\square=-1 \square \square=4$ ?

2.5 Determine los intervalos donde la función crece y decrece.

Fig. 1: Actividad de aprendizaje tradicional (AT) 
Instrucciones: Desarrolle cada uno de los problemas siguientes, cuide que su procedimiento sea claro y ordenado. Enmarque sus resultados finales.

1. Accese a cualquier dispositivo que le permita observar el video cuya liga es https://www.facebook.com/joseluisperezcastillo24/videos/1660875390851207 y responda:

1.1 ¿Cuántos litros de agua cree que se contaminaron en los 52 segundos que dura el video? (subraye la respuesta que considere)

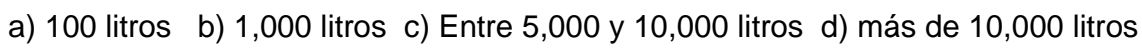

1.2 Para investigar la exactitud de los litros que se contaminaron evalúe la siguiente función para $x=5.2$

$$
\square(\square)=2,600+2 \square+100 \square^{2}
$$

Donde $\square(\square)$ mide los litros de agua contaminados y $\square$ son los segundos transcurridos después de iniciar la descarga de contaminantes, $\square$ está dada en decenas.

1.3 En base al modelo anterior halle e interprete $\square$ (1), $\square$ (3) $\square \square$ (4)

1.4 Grafique en un sistema de referencia los puntos anteriores y bosqueje el comportamiento para $\square(\square)$.

1.5 Tú, ¿cuidas el agua? Si es sí, responde ¿Qué acciones llevan a cabo tú y tu familia para cuidar el agua?

\section{Analizando la corrupción del país}

2.1 Investigue ¿qué es el índice de percepción de corrupción? y resuma su definición a dos renglones.

2.2 Los siguientes datos muestran el índice de percepción de corrupción que ha obtenido México en algunos años, entre el año 2002 al año $2016^{*}$, donde $\square=0$ corresponde al año 2000.

$(2,3.6),(4,3.6),(5,3.5),(6,3.3),(7,3.5),(8,3.6),(9,3.3),(10,3.1),(13,3.5),(14,3.7)$ y $(16,3.8)$

Con los datos anteriores modele una función con el menor error de aproximación, utilizando cualquier software y escriba la función hallada, llámele $\square(\square)$.

2.3 Determine con su modelo, cuál es el índice de percepción de corrupción que se espera tendremos este año.

2.4 Halle la primera derivada de la función obtenida y calcule e interprete $\square$ '(3), $\square$ '(10), $\square$ '(12).

2.5 ¿A qué velocidad cambió la percepción de corrupción en el año 2005 y 2006 ?

2.6 ¿En qué años (o intervalos de años) el índice de percepción de corrupción crece y en qué años decrece?

2.7 Defina con sus palabras qué es corrupción y describa cinco ejemplos, ya sea con dibujos propios, ilustraciones o fotos donde se aprecie este fenómeno.

2.8 Conteste ampliamente ¿La corrupción sólo es cuestión de política o de algún sector en específico?.

2.9 Tú, ¿combates la corrupción?

* Fuente: Transparency International, organización global de la sociedad civil que lidera la lucha contra la corrupción. www.transparency.org

Fig. 2: Actividad de aprendizaje contextualizada (AC)

Ambas actividades se implementaron por separado en dos grupos: el Grupo A integrado por 15 alumnos y el Grupo B por 22 alumnos. La AT se aplicó inicialmente en el Gpo. A, seguido por la AC. El orden de aplicación se invirtió para el Gpo. B. En ambos se evitó hacer mención alguna de las diferencias entre ellas, y se les informó del impacto equitativo en su calificación parcial. Respecto a la estructura, ambas actividades están conformadas por dos ejercicios. La AT tiene siete incisos, todos de contenido matemático. Por otro lado, la AC tiene ocho de contenido matemático y seis de contenido contextual. La Tabla 1 muestra la relación comparativa entre los reactivos de cada actividad, fundamentando su equivalencia en el conocimiento y/o habilidad requerida para responderlos.

Los ejercicios en ambas actividades de aprendizaje son problemas adecuados para el trabajo escolar. Para Van den Heuvel-Panhuizen (1999, citado en Beswick, 2011) un problema adecuado debería no ser ambiguo, proporcionar a los estudiantes todos los datos necesarios y tener sólo una respuesta correcta. La AT busca trabajar conceptos del tema de funciones y derivadas, mientras que la AC pretende ayudar a los estudiantes, además de practicar los mismos conceptos, a establecer vínculos entre los conceptos matemáticos y el mundo real. Esto implica desarrollar aprendizajes significativos y flexibles, los cuales según Beswick (2011) son aquellos que pueden utilizarse en cualquier contexto cuando sea necesario. En cuanto a la forma de presentación de las actividades en la AC: el Problema 1 parte de un video y el 2 con la introducción al concepto de índice de percepción de corrupción. Las preguntas 1.1, 1.5, 2.1, 2.7, 2.8 y 2.9 no se tomaron en cuenta para la calificación y serán parte de un estudio futuro para evaluar otro tipo de competencias. 
Tabla 1: Comparación de reactivos de la AT con los reactivos de la AC y su ubicación en Figura 5.

\begin{tabular}{|c|c|c|}
\hline $\begin{array}{c}\text { Reactivo de la Actividad } \\
\text { Contextualizada (AC) }\end{array}$ & $\begin{array}{c}\text { Reactivo equivalente en la Actividad } \\
\text { Tradicional (AT) }\end{array}$ & Representación en Figura 5 \\
\hline 1.1 & No hay equivalente & 2 \\
\hline 1.2 y 1.3 & 1.1 & No representado \\
\hline 1.4 & 1.2 & No representado \\
\hline 1.5 & No hay equivalente & 3 \\
\hline 2.1 & No hay equivalente & 5 \\
\hline 2.2 & 2.1 & 6 \\
\hline 2.3 & 2.2 & 7 \\
\hline 2.4 & 2.3 & No representado \\
\hline 2.5 & 2.4 & No representado \\
\hline 2.6 & 2.5 & No representado \\
\hline 2.7 & No hay equivalente & \\
\hline 2.8 & No hay equivalente & No hay equivalente \\
\hline 2.9 & & \\
\hline
\end{tabular}

Posterior a la aplicación de las actividades de aprendizaje, se aplicó un instrumento de tipo encuesta, titulado "Funciones y Derivadas", de seis ítems con respuestas delimitadas. Éstas fueron: 1) ¿Qué actividad te gustó más?, 2) ¿Qué actividad te causó mayor interés?, 3) ¿Qué actividad te proporciona información relevante para comprender tu entorno?, 4) ¿Qué actividad consideras contribuyó en mayor medida en tu formación personal?, 5) ¿Qué actividad consideras contribuyó en mayor medida en tu formación profesional?, 6) ¿Percibes que aprendiste más Matemáticas en una actividad que en la otra? Este instrumento fue analizado en forma descriptiva e inferencial.

\section{RESULTADOS}

Se presentan inicialmente los resultados que dan respuesta a las percepciones del sentido de utilidad de las matemáticas analizados a través de estadística descriptiva. Posteriormente se muestra la información para analizar las percepciones y desempeño en la AC con análisis descriptivo e inferencial.

\section{Análisis de las percepciones del sentido de utilidad de las Matemáticas}

Respecto a la utilidad de las Matemáticas en su formación profesional, las respuestas de los alumnos a la encuesta "Las Matemáticas en tu vida", se muestran en la Tabla 2. Los resultados de la encuesta "Temas de interés", se detallan por pregunta. El concentrado de respuestas a la primera pregunta se muestra en la Figura 3, manifestando las inquietudes de los universitarios, su contexto y preocupación por el entorno. La problemática social, económica y ecológica fueron los temas de mayor interés; en específico los fenómenos corrupción, pobreza y gasolinazo fueron los más mencionados por los estudiantes. Éste último hace referencia al descontento social vivido en México a partir del 1 de enero de 2017 por el aumento desproporcionado del precio de la gasolina.

Tabla 2: Resultados de percepción del sentido de utilidad de las Mat. en la formación profesional de estudiantes universitarios de áreas de Ciencias Sociales. Validación con Alfa de Cronbach de 0.7828.

\begin{tabular}{|c|c|c|c|}
\hline Ítem & Opciones de respuestas & Mat. I (\%) & Mat. II (\%) \\
\hline \multirow{4}{*}{$\begin{array}{c}\text { ¿Con cuál afirmación te } \\
\text { identificas más? }\end{array}$} & $\begin{array}{c}\text { Las Matemáticas no son muy útiles, ya } \\
\text { que sólo se necesita lo básico. }\end{array}$ & 7 & 16 \\
\cline { 2 - 4 } & $\begin{array}{c}\text { Imagino que las Matemáticas son útiles, } \\
\text { aunque no sabría dar un ejemplo de su } \\
\text { aplicación. }\end{array}$ & 46 & 25 \\
\cline { 2 - 4 } & $\begin{array}{c}\text { Las Matemáticas son muy útiles y podría } \\
\text { dar ejemplos de su aplicación. }\end{array}$ & 47 & 59 \\
\hline
\end{tabular}


Tabla 2: (Continuación)

\begin{tabular}{|c|c|c|c|}
\hline Ítem & Opciones de respuestas & Mat. I (\%) & Mat. II (\%) \\
\hline \multirow{2}{*}{$\begin{array}{c}\text { ¿Crees que tu carrera profesional } \\
\text { alcanzará los objetivos y } \\
\text { competencias si omitimos las } \\
\text { clases de Matemáticas? }\end{array}$} & Sí & 29 & 41 \\
\hline & No & 71 & 59 \\
\hline \multirow{2}{*}{$\begin{array}{l}\text { ¿Crees que las clases de } \\
\text { Matemáticas son o han sido un } \\
\text { obstáculo para que tú o alguno de } \\
\text { tus compañeros no termine sus } \\
\text { estudios profesionales? }\end{array}$} & $\mathrm{Si}$ & 66 & 63 \\
\hline & No & 34 & 37 \\
\hline \multirow{2}{*}{$\begin{array}{l}\text { ¿Crees recurrir a las Matemáticas } \\
\text { con frecuencia, al término de tu } \\
\text { formación profesional? }\end{array}$} & $\mathrm{Si}$ & 73 & 76 \\
\hline & No & 27 & 24 \\
\hline
\end{tabular}

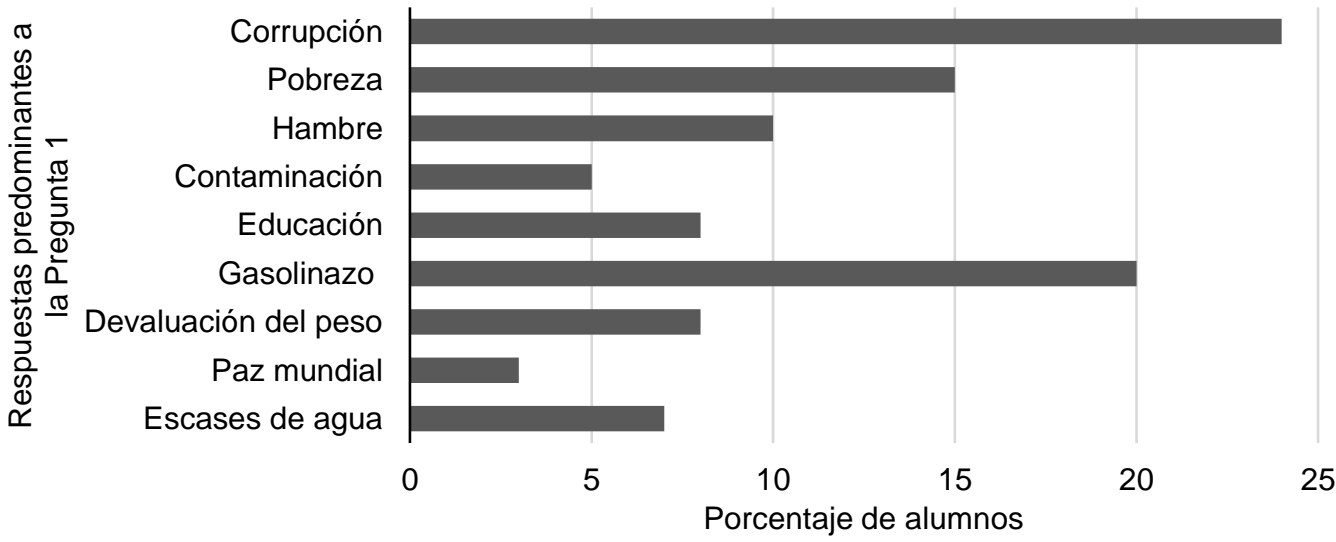

Fig. 3: Respuesta de los alumnos de Matemáticas para Ciencias Sociales a la interrogante "¿Qué problema quieres resolver?", como parte de la encuesta "Temas de interés".

Las respuestas a la Pregunta 2 "¿En qué te ayudan las Matemáticas para abordar y resolver esta problemática?" se reportan en la Figura 4. Estas se centraron, de acuerdo al análisis de las autoras, en cuatro vertientes: la primera, la mayoría de los alumnos mencionaron que las Matemáticas les ayudarán a hacer cálculos, sin mencionar más detalle respecto a ello o hacer mención de algún cálculo en específico. La segunda está integrada por afirmaciones como: "yo creo que no ayudan en nada" o similares a: "creo que otras materias ayudarían más, aquí no se necesitan las Matemáticas". La tercera se integró por: "no sé en qué me puedan ayudar", incluyendo desde aquellos que contestaron solo "no sé", hasta aquellos que especificaron: "sé que puede ayudar, pero no sé en qué o cómo". Finalmente, la cuarta, siendo el menor porcentaje, dijo que esta ciencia le ayudará a hacer cálculos para comprender el problema, sin especificar el tipo de cálculo a realizar.

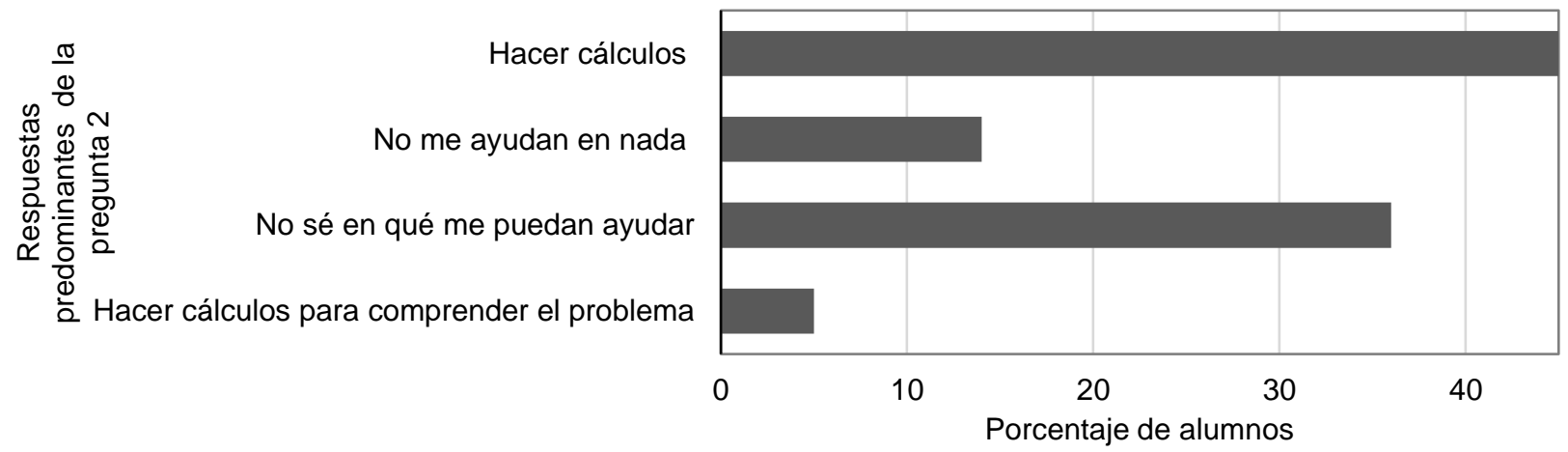

Fig. 4: Respuestas de los alumnos de Matemáticas para CS a la interrogante “¿En qué te ayudan las Matemáticas para abordar y resolver la problemática de tu interés?", como parte de la encuesta "Temas de interés". 


\section{Análisis del desempeño en las actividades de aprendizaje}

La evaluación de las actividades de aprendizaje se hizo por reactivo, otorgando una calificación de 1 o 0 para identificar respuestas correctas e incorrectas, respectivamente. Los resultados por reactivo en ambas actividades de aprendizaje, se muestran en la Figura 5A para el Grupo A y en la Figura 5B para el Grupo B.
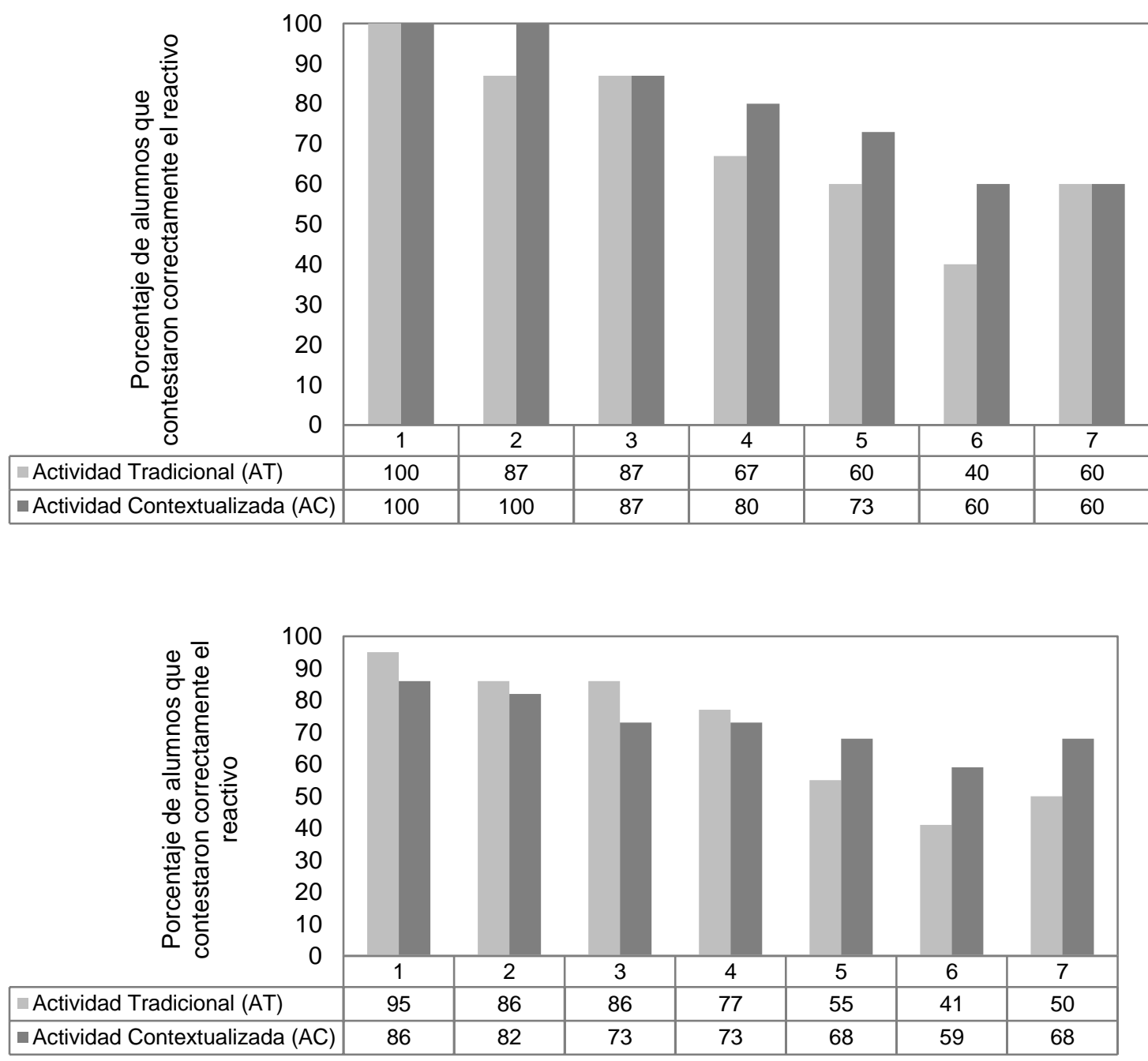

B)

Fig. 5: A) Desempeño del Grupo A al contestar reactivos equivalentes primero en la AT seguido por la AC. B) Desempeño del Grupo B al contestar reactivos equivalentes primero en la AC seguido por la AT. La identificación de los reactivos se encuentra en la Tabla 1.

\section{Análisis de las percepciones de las actividades de aprendizaje}

El instrumento titulado "Funciones y derivadas" permitió obtener información para conocer sus percepciones del material didáctico que resolvieron previamente. El concentrado de las respuestas a las primeras cinco preguntas se muestran en la Figura 6. Para el gusto e interés por las actividades de aprendizaje, ésta muestra predominio de la preferencia por la AC. En comparación con la Act. Tradicional, la Act. Contextualizada les gusto más al $76 \%$ de los estudiantes y al $73 \%$ les interesó más. Además, la perciben como detonante para comprender su entorno y favorecer la formación tanto personal como profesional, aunque esta última en menor medida.

En cuanto a la respuesta de los estudiantes a la pregunta: “¿Percibes que aprendiste más Matemáticas en una actividad que en la otra?", solamente el $51 \%$ dijo que sí. Estos resultados muestran que los estudiantes no perciben una diferencia significativa en su aprendizaje (apropiación de conceptos) de Matemáticas, pues el $49 \%$ no percibió haber aprendido más del tema de funciones y derivada con una actividad respecto a la otra. Para analizar la correlación entre las percepciones de las actividades de aprendizaje, se validaron hipótesis con la prueba estadística Chi cuadrada $\mathrm{X}^{2}$ a un nivel de confianza de $95 \%(\alpha=0.05)$ y con un Valor crítico de Chi cuadrada $\mathrm{V}_{\mathrm{CX} 2}=3.841$. Las hipótesis y resultados se muestran en la Tabla 3. 


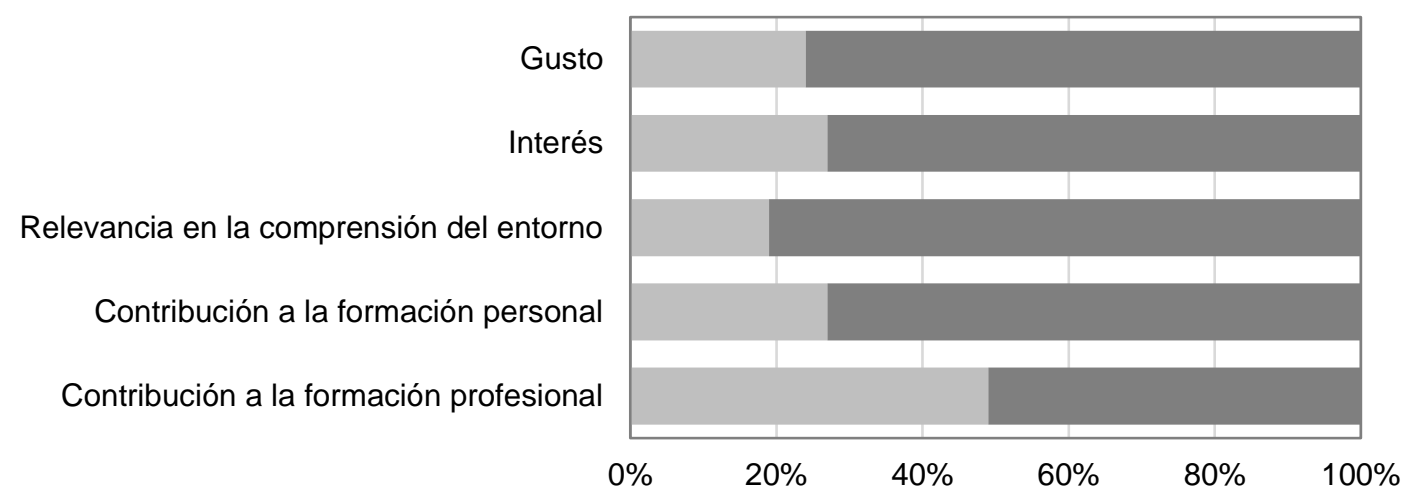

$\square$ Act. Tradicional $\square$ Act. Contextualizada

Fig. 6: Percepciones de los estudiantes después de haber resuelto las AT y AC. Resultados a las primeras cinco preguntas de la encuesta "Funciones y derivadas".

Tabla 3: Resultados de la correlación entre las variables gusto e interés por las actividades de aprendizaje y sentido de utilidad personal y profesional. Hipótesis validadas con la prueba estadística $\mathrm{X}^{2}$ a un nivel de confianza de $95 \%$ e instrumento validado con Alfa de Cronbach de 0.6029.

\begin{tabular}{|l|l|c|c|c|}
\hline \multicolumn{1}{|c|}{ Hipótesis Nula $H_{0}$} & Hipótesis alternativa $H_{1}$ & $X^{2}$ & $p$ & $H_{0}$ \\
\hline $\begin{array}{l}\text { No existe relación entre el gusto por } \\
\text { las actividades didácticas y el sentido } \\
\text { de utilidad personal }\end{array}$ & $\begin{array}{l}\text { Existe relación entre el gusto } \\
\text { por las actividades didácticas y } \\
\text { el sentido de utilidad personal }\end{array}$ & 1.829 & 0.176 & Aceptada \\
\hline $\begin{array}{l}\text { No existe relación entre el gusto por } \\
\text { las actividades didácticas y el sentido } \\
\text { de utilidad profesional }\end{array}$ & $\begin{array}{l}\text { Existe relación entre el gusto } \\
\text { por las actividades didácticas y } \\
\text { el sentido de utilidad profesional }\end{array}$ & 0.084 & 0.772 & Aceptada \\
\hline $\begin{array}{l}\text { No existe relación entre el interés por } \\
\text { las actividades didácticas y el sentido } \\
\text { de utilidad personal }\end{array}$ & $\begin{array}{l}\text { Existe relación entre el interés } \\
\text { por las actividades didácticas y } \\
\text { el sentido de utilidad personal }\end{array}$ & 12.830 & 0.00 & Rechazada \\
\hline $\begin{array}{l}\text { No existe relación entre el interés por } \\
\text { actividades didácticas y el sentido de } \\
\text { utilidad profesional }\end{array}$ & $\begin{array}{l}\text { Existe relación entre el interés } \\
\text { por las actividades didácticas y } \\
\text { el sentido de utilidad profesional }\end{array}$ & 14.460 & 0.00 & Rechazada \\
\hline
\end{tabular}

Las variables interés y sentido de utilidad, tanto personal como profesional, sí guardan relación. Por otro lado, la variable gusto por la actividad, no tiene relación con el sentido de utilidad asignado tanto al desarrollo profesional como personal.

\section{DISCUSIÓN}

A continuación se discuten los resultados encontrados por pregunta de investigación, intercalando a su vez algunas implicaciones para mejorar la práctica docente.

\section{Discusión sobre las percepciones del sentido de utilidad de las Matemáticas}

La respuesta a la primera pregunta de investigación se puede encontrar en la Tabla 2. Ahí se reporta que el $7 \%$ de los estudiantes de 10 semestre de las licenciaturas de ENH y EGCS no percibiendo la utilidad de las Mat.; mientras, el $34 \%$ no cree utilizarlas frecuentemente al egresar, e incluso el $66 \%$ de los encuestados la percibe como un obstáculo para la obtención de su grado. Estas percepciones sugieren el poco desarrollo de la competencia matemática de los estudiantes (Morales et al., 2010) y podría estar relacionado con su bajo desempeño (Zainal et al., 2017; Wan y Qiping, 2015; Altawallbeh et al., 2015, citados por Ayob y Yasin, 2017; Morales y García, 2013). La problemática se agudiza en el $2^{\circ}$ semestre, al aumentar el porcentaje del $29 \%$ al $41 \%$, del número de alumnos reconociendo el alcance de los objetivos y competencias en su carrera profesional incluso omitiendo las clases de Mat. Es decir, conforme avanzan en sus cursos de Mat., más se convencen de la poca aportación de la materia a su formación profesional, llegando incluso, el $63 \%$ de ellos a reconocerlos como un obstáculo para graduarse. Lo anterior sugiere la debilidad de las estrategias 
didácticas tradicionales implementadas en estos cursos, para contribuir en el fortalecimiento del sentido de utilidad, valoración y competencia matemática.

En cuanto al interés de los estudiantes, la Figura 3 evidencia su mayor preocupación en temas sociales, económicos y medioambientales, así como otros tópicos de su actualidad, por ejemplo, el gasolinazo en México. La omisión de las problemáticas científicas o tecnológicas en su lista de intereses podría estar relacionada con su área de formación profesional, en este caso Ciencias Sociales; sin embargo, esto no podría asegurarse. Esta identificación de tópicos de su interés es valiosa; pues tal como afirman Farias y Pérez (2010) si se conocen los intereses, se podrá motivar a los estudiantes y orientar correctamente el proceso de enseñanza-aprendizaje. Por otro lado, en la resolución de la problemática mencionada (Figura 4), el $45 \%$ de los estudiantes consideran a la Mat. como útil para hacer cálculos y el $36 \%$ no están seguros si ésta contribuirá en algo y cómo lo hará. Estos resultados coinciden con el análisis de la Tabla 2 y representan un bajo porcentaje de alumnos que visualizan a esta ciencia como una herramienta valiosa en la resolución de los problemas de su interés, reflejando su baja competencia mat.

\section{Discusión sobre el desempeño y las percepciones en una actividad de aprendizaje contextualizada}

Al comparar las actividades tradicional (AT) y contextualizada (AC), ésta última se muestra más enriquecida, en cantidad de reactivos, carga visual y reflexión requerida; es decir, incluye problemas o preguntas para las cuales no existe un algoritmo previamente definido. Estas características implican mayor esfuerzo del profesor dedicado tanto al diseño como a la evaluación; sin embargo, podría conllevar otros beneficios mayores (Vid infra). Además, la elección del contexto entre los temas de interés mencionados por los estudiantes (Figura 3), hace que la AC cumpla la característica establecida por Beswick (2011) al referirse a un problema contextualizado como aquel con significado, propósito y dirigido al logro de un objetivo.

Por otra parte, la Figura 5 reporta el desempeño de los estudiantes por reactivo, en ambos grupos. Observando una pequeña mejora de hasta el 13\% en los reactivos 1 a 4 . Esto con certeza puede atribuirse a la práctica y no al contexto, ya que el orden de aplicación de las actividades no fue el mismo en ambos grupos. Es decir, el Grupo A, quien aplicó primero la AT, tuvo una mejora en la AC. Mientras el Grupo B, quien aplicó primero la AC, tuvo una mejora en la AT. Por el contrario, donde sí se observaron mejoras más significativas, de hasta $30 \%$, fue en los reactivos 5,6 y 7 , relacionados con los intervalos de crecimiento y decrecimiento. Esta mejora fue independiente del orden de aplicación; es decir, puede deberse al contexto, el cual favorece la interpretación intuitiva debida al significado. Lo anterior coincide con lo reportado por Beswick (2011), quien reconoce el uso de problemas contextualizados como un camino para mejorar la comprensión de conceptos matemáticos. Esta discrepancia merece mayor investigación y análisis; así mismo el desempeño de los estudiantes en aquellas preguntas en la AC sin equivalente en la AT, las cuales serán evaluadas en términos del desarrollo de otras competencias en futuras publicaciones de las autoras.

Respecto a las percepciones estudiantiles sobre las actividades aplicadas, la Figura 6 muestra mayor agrado e interés por la actividad contextualizada respecto a la tradicional. Esto reitera que la elección del tema de la AC fue correcta en cuanto al significado de un contexto según Beswick (2011). Además, la AC es identificada como la de mayor relevancia para la comprensión del entorno y contribución en la formación personal. Sin embargo, en su contribución a la formación profesional ambas son consideradas igualmente valiosas. Esto podría estar relacionado con la percepción habitual y más limitada de las Mat. en el ámbito escolar, la cual según Reid et al. (2005) se enfoca al aprendizaje de técnicas. Es decir, el alumno no está acostumbrado a ver a las Mat. en los contextos mostrados en la AC y quizá dude si impactará en su formación profesional. Por otro lado, la Figura 6 evidencia la escasa percepción de los alumnos por un mayor aprendizaje en una actividad respecto a la otra. Ésta no es una problemática a tratar en la presente investigación, porque el objetivo es analizar el sentido de utilidad de las Matemáticas en Actividades Tradicionales y en Actividades Contextualizadas.

La validación de la relación entre las variables interés y gusto por las actividades de aprendizaje y el sentido de utilidad personal y profesional, apoya en la respuesta a la segunda pregunta de investigación planteada. El interés por las actividades de aprendizaje sí tiene relación con el sentido de utilidad, tanto personal como profesional, no así el gusto. Esto confirma que el material didáctico presentado a los estudiantes efectivamente fomenta su interés y con ello impacta en el sentido de utilidad de la Mat. para su desarrollo profesional. Esto coincide con lo reportado en la literatura, afirmando que las características de la tarea solicitada a los alumnos determinan el interés y la motivación intrínseca (Ayob y Yasin, 2017; Farias y Pérez, 2010). Beswick (2011) menciona cinco propósitos para usar problemas contextuales, cuyo logro se discutirá a continuación. El primero es satisfacer las necesidades económicas de la sociedad. Éste se cumple, pues la AC está enmarcada en la Educación Matemática Crítica (EMC), un proceso abierto funcional aplicable de distintas maneras según los factores considerados, por ejemplo, "educar para los negocios", "educar al consumidor", "educar al trabajador", "educar para la justicia social", entre otros; que fusiona a las Matemáticas 
y su enseñanza con la finalidad de ponerlas al servicio de una ciudadanía ilustrada y crítica (Sáenz y García, 2015). El segundo tiene la intención de mejorar la comprensión de temas importantes y se logró durante el diseño de la AC; pues se diseñó a partir de temas relevantes para los estudiantes, fomentando así su comprensión a través de la Mat. El tercer propósito es mejorar la comprensión de conceptos matemáticos y su logro merece trabajo de investigación a futuro, tal como se discutió en el análisis de la Figura 5. Cuarto, la apreciación de la naturaleza de las Matemáticas también se hizo presente; aunque en su nivel más extendido y limitado al manejo de técnicas (Reid et al., 2005), pues los alumnos no reconocen la reflexión y el análisis como competencia matemática. Sin embargo, al menos el $50 \%$ de los estudiantes ya consideran a la AC como fuente de contribución a su formación profesional (supra cit.). Esto es muy relevante y debe seguirse trabajando desde las instituciones, los diseños curriculares y la práctica docente cotidiana. El último propósito mencionado por Beswick (2011) es mejorar el afecto por las Mat. Éste debe investigarse con mayor profundidad para validar su correlación con el tipo de actividades de aprendizaje.

El diseño y evaluación de actividades didácticas contextualizadas puede requerir mayor trabajo y dedicación por parte de los docentes; sin embargo, los beneficios alcanzados en el mejoramiento de la competencia matemática son muy valiosos. Además, el contexto no es una técnica didáctica, es decir no está encajonada a la aplicación de una pedagogía específica, más bien es una estrategia efectiva de enseñanza-aprendizaje que debe fomentarse desde los currículos y desde las instituciones.

\section{CONCLUSIONES}

Ante los resultados cuantitativos obtenidos y su discusión, se llegaron a las siguientes conclusiones relevantes: A) Los estudiantes universitarios del área de Ciencias Sociales que cursan las Materias de Matemáticas I y II mostraron un escaso sentido de utilidad de los cursos de Mat. en su actividad profesional, identificándolos incluso como un obstáculo para la obtención del grado. Esta percepción se agravó al pasar del primero al segundo semestre. B) Los tópicos de su interés fueron las problemáticas sociales, económicas y medioambientales, preferentemente de actualidad. Por lo que las actividades de aprendizaje contextualizadas deben diseñarse partiendo de estas temáticas. C) Al contrastar el desempeño de los estudiantes en una actividad contextualizada respecto a una respecto actividad tradicional, se observó que su interpretación de un concepto matemático mejoró al estar inmersa en un contexto; mientras que en las repeticiones de algoritmos el contexto no mostró beneficios relevantes. D) Los estudiantes percibieron con mayor interés y sentido de utilidad a la actividad contextualizada por encima de la actividad tradicional, fomentando su competencia matemática.

\section{AGRADECIMIENTOS}

Las autoras reconocen el apoyo técnico y financiero de Writing Lab, TecLabs, Tecnológico de Monterrey, México, en la producción de este trabajo.

\section{REFERENCIAS}

Altawallbeth, M., F. Soon, W. Thiam y S. Alshourah, Mediating Role of Attitude, Subjective Norm and Perceived Behavioural Control in the Relationships between their Respective Salient Beliefs and Behavioural Intention to Adopt Elearning among Instructors in Jordanian Universities, Journal of Education and Practice, Tomado de Ayob y Yasin (2017)

Ayob, A. y R.M. Yasin, Factors Affecting Attitudes Towards Mathematics, doi: 10.6007/IJARBSS/v7-i11/3548, International Journal of Academic Research in Business and Social Sciences, 7(11), 1100-1109, (2017)

Azcárate, C., M. Camacho-Machín, M.T. González, y M. Moreno, (Coords.) Didáctica del Análisis Matemático: Una revisión de las investigaciones sobre su enseñanza y aprendizaje en el contexto de la SEIEM, $1^{\underline{a}}$ Ed., 19-63, Servicio de publicaciones Universidad de la Laguna, Santa Cruz de Tenerife, España, (2015)

Beswick, K., Putting Context in Context: An Examination of the Evidence for the Benefits of "Contextualised" Tasks, doi: 10.1007/s10763-010-9270-z, International Journal of Science and Mathematics Education, 9, 367-390, (2011)

Cooper, B. y T. Harries, Children's Responses to Contrasting 'Realistic' Mathematics Problem: Just how Realistic are Children ready to be? Education Studies in Mathematics, Tomado de Beswick (2011)

Dickinson, P. y S. Hough, Using Realistic Mathematics Education in UK Classrooms, ISBN: 978-0-948186-24-0, 1-26, Centre for Mathematics Education, Manchester Metropolitan University, Manchester, Uk, (2012)

Farias, D. y J. Pérez, Motivación en la Enseñanza de las Matemáticas y la Administración, doi: 10.4067/S071850062010000600005, Formación Universitaria, 3(6), 33-40, (2010)

Gravemeijer, K., M. Stephan y otros tres autores, What Mathematics Education May Prepare Students for the Society of the Future? DOI: 10.1007/s10763-0176-017-9814-6, Int J of Sci and Math Educ, 15(1), 105-123, (2017)

Gutiérrez, A. (Ed.), Área de conocimiento didáctica de la Matemática, 11-26, Editorial Síntesis, Madrid, España (1999) 
Hernández, R., C. Fernández y P. Batista, Metodología de la investigación, México, México, McGraw Hill Education, (2014) Jurdak, M.E. Contrasting Perspectives and Performance of High School Students on Problem Solving in Real World Situated, and School Contexts, Educational Studies in Mathematics, Tomado de Beswick (2011)

Kline, M., El fracaso de la matemática moderna, 12a Ed., 8-30, Siglo XXI editores, México, México, (1986)

Morales, L.M., J.G. Sánchez y H. Roldán, Influencia de la Actitud en el Rendimiento Académico en Matemáticas con Estudiantes Universitarios, Acta Latinoamericano de Matemática Educativa, Comité Latinoamericano de Matemática Educativa A. C.,455-463, México, D.F., (2010)

Morales, L.M. y O.E. García, La Afectividad de la Inteligencia, doi: 10.4067/S0718-50062013000500002, Formación Universitaria, 6(5), 3-12, (2013)

Osborne, J., S. Simon y S. Collins, Attitudes towards Science: A Review of the Literature and its Implications, DOI: 10.1080/0950069032000032199, International Journal of Science Education, 25(9), 1049-1079, (2003)

Palacios, A., V. Arias y B. Arias, Las Actitudes hacia las Matemáticas: Construcción y Validación de un Instrumento para su Medida, doi: 10.1387/RevPsicodidact.8961, Revista de Psicodidáctica, 19(1), 67-91, (2014)

Perry, C.A., Motivation and Attitude of Preservice Elementary Teachers toward Mathematics, Morehead State University, Tomado de Palacios, Arias y Arias (2014)

Reid, A., L.N. Wood, G.H. Smith y P. Petocz, Intention, Approach and Outcome: University Mathematics Students' Conceptions of Learning Mathematics, doi: 10.1007/s10763-004-5818-0, International Journal of Science and Mathematics Education, 3, 567-586, (2005)

Ruiz, M., La respuesta educativa ante la formación de las competencias profesionales y laborales, Profesionales

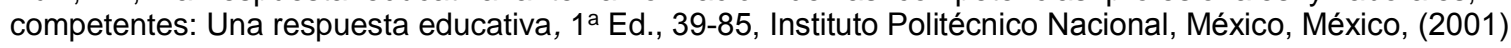

Sáenz, C. y X. García, Elementos de una educación matemática crítica, Matemáticas Placer, Poder, a veces dolor: Una mirada crítica sobre la matemática y su enseñanza, UAM Ediciones, 223-255, Madrid, España, (2015)

Serrano, J.M., M.E. González-Herrero y R.M. Pons, Aprendizaje cooperativo en Matemáticas, 1ํㅡㄹ Ed., 69-86, editum Ediciones de la Universidad de Murcia, Murcia, España, (2008)

Skovsmose, O., An Invitation to Critical Education, Nueva York: Springer, Tomado de Sáenz y García (2015)

Tan, S.T., Matemáticas aplicadas a la administración, la vida y las Ciencias Sociales, Cengage Editores, (2015)

Van den Heuvel-Panhuizen, M., Context problems and assessment: Ideas from the Netherlands; in Issues in teaching numercy in primary schools by I. Thomson (Ed.), pp 130-142, Open University Press, Maidenhead, UK, (1999)

Wan, C. y S.G. Qiping, Encouraging the Use of Urban Green Space: The Mediating Role of Attitude, Perceived Usefulness and Perceived Behavioural Control, Habitat International, Tomado de Ayob y Yasin (2017)

Wood, L.N., G. Mather y otros siete autores, University Students' Views of the Role of Mathematics in their Future, doi: 10.1007/s10763-011-9279-y, International Journal of Science and Mathematics Education, 10, 99-119, (2012)

Zainal, N.T., A. Harum y J. Lili, Examining the Mediating Effect of Attitude towards Electronic Words-of Mouth (eWOM) on the Relation between the Trust in eWOM Source and Intention to follow eWOM among Malaysian Travellers, Asia Pacific Management Review, Tomado de Ayob y Yasin (2017)

Zemelman, S., D. Harvey y A. Hayde, Best Practice: New Standards for Teaching and Learning in America's Schools, Tomado de Farias y Pérez (2010) 